HD-THEP-00-02

WUE-ITP-2000-007

\title{
Learning structured data from unspecific reinforcement
}

\author{
Michael Biehl ${ }^{1}$, Reimer Kühn², Ion-Olimpiu Stamatescu ${ }^{2,3}$ \\ ${ }^{1}$ Institut für Theoretische Physik, Universität Würzburg, Am Hubland \\ D-97074 Würzburg, Germany \\ ${ }^{2}$ Institut für Theoretische Physik, Universität Heidelberg, Philosophenweg 16 und 19 \\ D-69120 Heidelberg, Germany \\ ${ }^{3}$ Forschungstätte der Evangelischen Studiengemeinschaft, Schmeilweg 5, \\ D-69118 Heidelberg, Germany
}

October 30, 2018

\begin{abstract}
We show that a straightforward extension of a simple learning model based on the Hebb rule, the previously introduced Association-Reinforcement-Hebb-Rule, can cope with "delayed", unspecific reinforcement also in the case of structured data and lead to perfect generalization.
\end{abstract}

\section{Introduction}

Learning from unspecific reinforcement may be essential in various contexts, both natural and artificial, where, typically, the results of particular actions add to a final consequence which only is valuated. The freedom residing in each step is not (or only partially) controlled directly and the learner must cope with the necessity of improving its performance only from information concerning the final success of a complex series of actions.

It is therefore important to find out whether there are simple and robust procedures for such situations, which might have developed under natural conditions and which may be basic also for artificial learning rules (for this reason we do not consider evolved algorithms like Q-learning [1], TD learning [2] etc). In previous works [3], [4] we have introduced an "Association-Reinforcement" learning model based on the following conception:

1. For each given input (external situation) the agent answers with an action (operation) depending solely on the input and on its instantaneous internal (cognitive) structure and simultaneously strengthens (in its internal structure) the blind association between this particular input and action.

2. At the end of a series of actions (path) the final success is judged. Then the associations "situation - operation" which have been involved on this path are re-weighted equally and depending only on the final success - unspecific reinforcement. 
In [4] we studied an implementation of this model to a classification problem for perceptrons, the Association-Reinforcement-Hebb-rule and showed some amazing properties:

a) Despite the fact that feedback on the learner's performance enters its learning dynamics only in an unspecific way in that it cannot be associated with single identifiable correct or incorrect associations, convergence of the AR-Hebb-algorithm in the sense of asymptotically perfect generalization is found.

b) For given initial conditions, this convergence depends on the learning parameters characterizing the 2 steps described above; in particular none of these steps can be completely inhibited. Alternatively, for given algorithm parameters convergence may depend on initial conditions.

In detail the dynamics of this algorithm was found to be very complex and interesting, being controlled by fixed points in the pre-asymptotic regime, and having a continuous set of asymptotic convergence laws. These results could easily be extended to the more realistic case where in the second step the unspecific reinforcement is randomly applied to only part of the associations achieved in the first step (the agent does not recall everything it has done on the trial) [4]. Further interesting extensions concern the question of structured data and of multilayer perceptrons.

Structured data represent a more involved classification problem and it is known that when teacher and data vector are not fully aligned (or exactly uncorrelated) the usual Hebb rule does not lead to convergence of the student vector onto that of the teacher, while the perceptron algorithm does [5]. On the other hand, the limiting case of the AR-Hebb-rule corresponding to the perceptron rule has been shown not to converge in the case of unspecific reinforcement for non-structured data. It is therefore a non-trivial question whether the unspecific reinforcement problem can be solved for structured data and in particular, whether some immediate extension of the AR-Hebb-rule can be shown to converge in this case. It is this question which we shall address in this paper. In a future publication we shall treat the problem of the committee machine as a first step to multi-layer perceptrons.

In section 2 we shall describe the learning model in the general setting and in section 3 we shall discuss its convergence properties providing numerical and analytical results. Thereby we shall briefly recall the non-structured data case and then concentrate on the general, structured data case. Section 4 contains the conclusions.

\section{Learning rule for perceptrons under unspecific reinforcement}

We consider one layer perceptrons with Ising or real number units $s_{i}$, real weights (synapses) $J_{i}$ and one Ising output unit:

$$
s=\operatorname{sign}\left(\frac{1}{\sqrt{N}} \sum_{i=1}^{N} J_{i} s_{i}\right)
$$

Here $N$ is the number of input nodes, and we put no explicit thresholds. The network (student) is presented with a series of patterns $s_{i}=\xi_{i}^{(q, l)}, q=1, \ldots, Q, l=1, \ldots, L$ to which it answers with $s^{(q, l)}$. A training period consists of the successive presentation of $L$ patterns. The answers are compared with the corresponding answers $t^{(q, l)}$ of a teacher with pre-given weights $B_{i}$ and the 
average error made by the student over one training period is calculated:

$$
e_{q}=\frac{1}{2 L} \sum_{l=1}^{L}\left|t^{(q, l)}-s^{(q, l)}\right|
$$

The training algorithm consists of two parts:

I. - a "blind" Hebb-type association at each presentation of a pattern:

$$
J_{i}^{(q, l+1)}=J_{i}^{(q, l)}+\frac{a_{1}}{\sqrt{N}} s^{(q, l)} \xi_{i}^{(q, l)}
$$

II. - an "unspecific" but graded reinforcement proportional to the average error $e_{q}$ introduced in (2), also Hebbian, at the end of each training period,

$$
J_{i}^{(q+1,1)}=J_{i}^{(q, L+1)}-\frac{a_{2}}{\sqrt{N}} e_{q} \sum_{l=1}^{L} r_{l} s^{(q, l)} \xi_{i}^{(q, l)} .
$$

where $e_{q}$ is the average error eq. (2) and $r_{l}$ is a dichotomic random variable:

$$
r_{l}=\left\{\begin{array}{lll}
1 & \text { with probability } & w \\
0 & \text { with probability } & 1-w
\end{array}\right.
$$

Because of these 2 steps we called this algorithm "association/reinforcement(AR)-Hebb-rule". We are interested in the behavior with the number of iterations $q$ of the generalization error $\epsilon_{g}(q)$ :

$$
\epsilon_{g}(q)=\frac{1}{\pi} \arccos \left(\frac{\boldsymbol{J} \cdot \boldsymbol{B}}{|\boldsymbol{J}||\boldsymbol{B}|}\right),
$$

in particular we shall test whether the behavior of $\epsilon_{g}(q)$ follows a power law at large $q$ :

$$
\epsilon_{g}(q) \simeq \text { const } q^{-p}
$$

The training patterns $\left\{\xi_{i}^{(q, l)}\right\}$ are generated randomly from the following distribution:

$$
\begin{aligned}
P(\boldsymbol{\xi}) & =\frac{1}{2} \sum_{\sigma= \pm 1} P(\boldsymbol{\xi} \mid \sigma) \\
P(\boldsymbol{\xi} \mid \sigma) & =\prod_{i=1}^{N} \frac{1}{\sqrt{2 \pi}} \mathrm{e}^{-\frac{1}{2}\left(\xi_{i}-m \sigma C_{i}\right)^{2}}
\end{aligned}
$$

and we take:

$$
\boldsymbol{C}^{2}=\boldsymbol{B}^{2}=N, \quad \boldsymbol{C} \cdot \boldsymbol{B}=\eta N
$$

with fixed, given $m, \eta$. Notice the following features: 
a) During training the student only uses its own associations $\boldsymbol{\xi}^{(q, l)} \leftrightarrow s^{(q, l)}$ and the average error $e_{q}$ which does not refer specifically to the particular steps $l$.

b) Since the answers $s^{(q, l)}$ are made on the basis of the instantaneous weight values $\boldsymbol{J}^{(q, l)}$ which change at each step according to eq. (3), the series of answers form a correlated sequence with each step depending on the previous one. Therefore $e_{q}$ measures in fact the performance of a "path", an interdependent set of decisions.

c) In contrast with the case studied in [4] the patterns can now have a structure. This introduces essential differences to the previous situation, as we shall see in the next section.

d) We explicitly account for imperfect recall at the reinforcement step by the parameter $w$ (5). This introduces a supplementary, biologically motivated randomness which, as already suggested in [4], does not appear to introduce qualitative changes in the results, however (see section 3 ).

e) For $L=1$ (and $w=1$ ) the algorithm reproduces the usual "perceptron rule" (for $a_{1}=0$ ) or to the usual "unsupervised Hebb rule" (for $a_{2}=2 a_{1}$ ) for on-line learning, for which the corresponding asymptotic behavior is known [6], [7], [5].

To study the learning behaviour we use Monte Carlo simulation and coarse grained analysis. The latter is provided by combining the blind association (3) during a learning period of $L$ elementary steps and the graded unspecific reinforcement (四) at the end of each learning period into one coarse grained step

$$
\begin{aligned}
J_{i}^{(q+1,1)} & =J_{i}^{(q, 1)}+\frac{1}{\sqrt{N}}\left(a_{1}-a_{2} e_{q}\right) \sum_{l=1}^{L} r_{l} \operatorname{sign}\left(\frac{1}{\sqrt{N}} \sum_{j=1}^{N} J_{j} \xi_{j}^{(q, l)}\right) \xi_{i}^{(q, l)} \\
e_{q} & =\frac{1}{2 L} \sum_{l=1}^{L}\left|\operatorname{sign}\left(\frac{1}{\sqrt{N}} \sum_{k=1}^{N} J_{k} \xi_{k}^{(q, l)}\right)-\operatorname{sign}\left(\frac{1}{\sqrt{N}} \sum_{i=k}^{N} B_{k} \xi_{k}^{(q, l)}\right)\right| .
\end{aligned}
$$

For simplicity we shall take for the time being $r_{l}=1$, i. e. $w=1$ in eq. (5). We introduce

$$
\alpha=q L / N, \quad \lambda=a_{1} / a_{2}
$$

and rescale everything with $a_{2}$, which means that we can take without loss of generality $a_{2}=1$ in (10), (11). We define the overlaps:

$$
\mathcal{R}(\alpha)=\frac{1}{N} \boldsymbol{B} \cdot \boldsymbol{J}^{(q, l)}, \mathcal{Q}(\alpha)=\frac{1}{N}\left[\boldsymbol{J}^{(q, l)}\right]^{2} \quad, \mathcal{D}(\alpha)=\frac{1}{N} \boldsymbol{C} \cdot \boldsymbol{J}^{(q, l)} .
$$

Note that in the "thermodynamic limit" $L / N \rightarrow 0$ the overlaps are self-averaging and we can neglect the dependence of $\mathcal{R}, \mathcal{D}$ and $\mathcal{Q}$ on $l$. We shall follow standard procedures [7], [8], [9], [10]. Treating $\alpha$ as a continuous variable and using:

$$
\begin{aligned}
x & \equiv \pi \epsilon_{g}=\arccos \left(\frac{\mathcal{R}}{\sqrt{\mathcal{Q}}}\right), \\
y & \equiv \arccos \left(\frac{\mathcal{D}}{\sqrt{\mathcal{Q}}}\right), \\
z & \equiv \arccos \eta
\end{aligned}
$$


we obtain the coarse grained equations:

$$
\begin{aligned}
\frac{d \mathcal{R}}{d \alpha}= & \frac{d}{d \alpha}(\sqrt{\mathcal{Q}} \cos x)=\left(\lambda-\frac{1}{2}\right) A_{J T}+\frac{1}{2 L} A_{T T}+\frac{1}{2}\left(1-\frac{1}{L}\right) S_{J T} A_{J T}, \\
\frac{d \mathcal{D}}{d \alpha}= & \frac{d}{d \alpha}(\sqrt{\mathcal{Q}} \cos y)=\left(\lambda-\frac{1}{2}\right) A_{J C}+\frac{1}{2 L} A_{T C}+\frac{1}{2}\left(1-\frac{1}{L}\right) S_{J T} A_{J C}, \\
\frac{d \sqrt{\mathcal{Q}}}{d \alpha}= & \left(\lambda-\frac{1}{2}\right) A_{J J}+\frac{1}{2 L} A_{T J}+\frac{1}{2}\left(1-\frac{1}{L}\right) S_{J T} A_{J J}+ \\
& \frac{1}{2 \sqrt{\mathcal{Q}}}\left[\left(\lambda-\frac{1}{2}\right) S_{J T}+\frac{1}{4}\left(1-\frac{1}{L}\right) S_{J T}^{2}+\left(\lambda-\frac{1}{2}\right)^{2}+\frac{1}{4 L}\right],
\end{aligned}
$$

where the expectation values $A, S$. are given in Appendix (section 5.1). These equations describe the flow of the three quantities $\epsilon_{g}=x / \pi, \mathcal{Q}$ and $y$ with $\alpha$ and involve the data/teacher parameters $m$ and $z=\operatorname{arcos} \eta$ and the learning parameter $\lambda$. Note the geometric constraint:

$$
\sin \frac{y}{2}-\sin \frac{z}{2}=\omega \sin \frac{x}{2}, \quad|\omega| \leq 1
$$

\section{Convergence behaviour of the AR-Hebb algorithm}

\subsection{Non-structured data}

The case of non-structured data $-m=0$ in eq. (35)-(43) - has been treated in [4], here we briefly recall some of the results for the later comparison with the structured data case.

Monte Carlo simulations indicate that in spite of the partial information contained in the unspecific reinforcement perfect generalization is achieved by the AR-Hebb algorithm and it depends on the learning parameters - see 沺. This intriguing behaviour is elucidated by the coarse grained analysis. In this case eqs. (17)-(19) reduce to two equations (for $\mathcal{R}$ and $\mathcal{Q}$ ) which have as general asymptotic solutions

$$
\begin{aligned}
\epsilon_{g}^{2} & \simeq \frac{1}{2 \pi\left(\frac{1}{\lambda L}-1\right)} \alpha^{-1}+\tilde{c}_{1} \alpha^{-\frac{1}{\lambda L}} \quad \text { for } \lambda \neq \frac{1}{L}, \\
\epsilon_{g}^{2} & \simeq\left(\frac{1}{2 \pi} \ln \alpha+\tilde{c}_{2}\right) \alpha^{-1} \quad \text { for } \lambda=\frac{1}{L}, \\
\mathcal{Q} & \simeq \frac{2}{\pi} \lambda^{2} \alpha^{2}
\end{aligned}
$$

at large $\alpha$. We see that for $\lambda<\frac{1}{L}$ we obtain asymptotically perfect generalization, the dominant term exhibiting the usual power $-1 / 2$, while for $\lambda>\frac{1}{L}$ the second term in (21) dominates and ensures again perfect generalization but with a different power law, $-1 /(2 \lambda L)$. For $\lambda=\frac{1}{L}$ we obtain logarithmic corrections - see eq. (22). Notice that these results hold also for $L=1$. One can generally see that for $\lambda=0$ one cannot have perfect generalization for $L>1$. For $L=1$ one re-obtains the asymptotic behavior found in [6].

This learning algorithm is further characterized by highly interesting pre-asymptotics, dominated by two stationarity conditions, one for the self-overlap, $d \mathcal{Q} / d \alpha=0$, and one for the 
generalization error $d \epsilon_{g} / d \alpha=0$. For suitable values of the network parameters, the two stationarity conditions may simultaneously be satisfied, leading to fixed points of the learning dynamics, one of them stable and of poor generalization, the other with one attractive and one repulsive direction. Correspondingly, the flow is divided by a separatrix defined by a critical $\lambda_{c}\left(\mathcal{Q}_{0}\right)$ into trajectories leading to convergence according to the asymptotic behaviour (21)-(23) for $\lambda>\lambda_{c}\left(\mathcal{Q}_{0}\right)$, or to poor generalization otherwise.

The salient features of these results for the case of non-structured data are the convergence of the AR-Hebb-algorithm in the sense of asymptotically perfect generalization with a power law depending on the learning parameters $L$ and $\lambda$ and the existence of a minimal value $\lambda_{c}\left(\mathcal{Q}_{0}\right)$, fixed by the pre-asymptotic structure and below which the system is driven toward complete confusion. Notice also that the best convergence is achieved for $\lambda$ just above $\lambda_{c}$. One last point concerns the recalling parameter $w$, eqs. (四),(阿). A rough first quantitative characterization of this modification would be that it leads to an effective rescaling of the parameter $\lambda$, viz. $\lambda \rightarrow \lambda / w$, leading to a corresponding reduction of critical $\lambda$ 's by approximately a factor $p$. This is well supported by numerical simulations (see also Fig. 11 for the case of structured data) and we conclude that the algorithm is stable against this supplementary element of indeterminism.

\section{$3.2 \quad$ Structured data}

Numerical simulations indicate that for $m \neq 0$ and $0<|\eta|<1$ the behaviour of the algorithm for all $w$ is more involved: generically, no convergence is found in this case for fixed values of the learning parameters. This agrees with the expectations, since, on the one hand the situation found at $L=1, w=1$ for structured data [6] could be expected to hold for every $L$, namely that Hebb updating leads to a nonzero asymptotic generalization error. On the other hand, the situation found before for non-structured data should hold also for structured data, namely that the perceptron rule $(\lambda=0)$ (which for $L=1$ was shown to lead to convergence also in the structured data case [6]) does not work for $L>1$.

In fact one can make a more general argument that for fixed $\lambda$ the AR-Hebb rule does not lead to perfect generalization for generically structured data. To obtain good generalization requires $\mathcal{R} / \sqrt{\mathcal{Q}} \rightarrow 1$ and $\mathcal{D} / \sqrt{\mathcal{Q}} \rightarrow \eta$, from which one may obtain the necessary dominant scaling (with $\mathcal{Q})$ of the various integrals appearing in (35)-(43), namely

$$
\begin{aligned}
& A_{J T} \simeq \kappa, \\
& A_{T J} \simeq \sqrt{Q} \kappa+o(\sqrt{Q}), \\
& A_{J J} \simeq \sqrt{Q} \kappa+o(\sqrt{Q}), \\
& A_{J C} \simeq \kappa, \\
& A_{T C} \simeq \kappa, \\
& A_{T T} \simeq \kappa, \\
& S_{J T} \simeq 1,
\end{aligned}
$$

with

$$
\kappa=m \eta \varphi(m \eta)+\sqrt{\frac{2}{\pi}} \mathrm{e}^{-m^{2} \eta^{2} / 2} .
$$




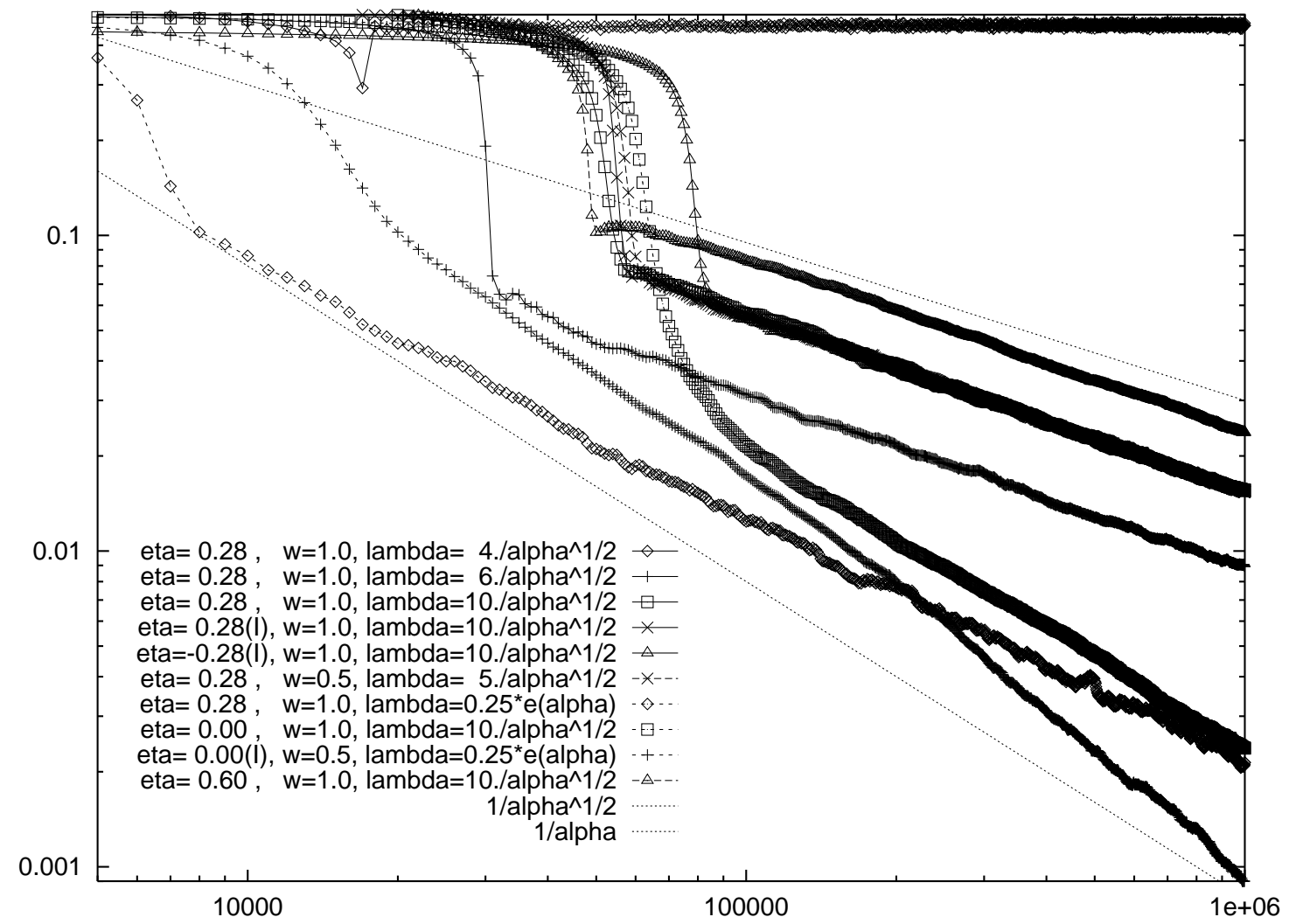

Figure 1: Generalization error $\epsilon_{g}$ vs. $q=\alpha N / L$ for $L=10, N=100$, various overlaps $\eta$ and starting point $\sqrt{\mathcal{Q}(0)}=100$. We use either patterns with $\xi_{i}$ : real numbers or $\xi_{i}$ : Ising spins (I) and $\lambda=\lambda_{0} / \sqrt{\alpha}$ or $\lambda=\lambda_{0} e(\alpha) / e(1)$, see (34). $w$ is the recall probability (4), (5). Note the change in behaviour between $\lambda_{0}=4$ and $\lambda_{0}=6$. The straight lines are illustrative power laws.

This in turn would lead to the following asymptotic expressions for the flow equations (17)-(19) (at fixed $\lambda$ )

$$
\begin{aligned}
& \frac{d \mathcal{R}}{d \alpha} \simeq \kappa \lambda, \\
& \frac{d \mathcal{D}}{d \alpha} \simeq \kappa \lambda, \\
& \frac{d \mathcal{Q}}{d \alpha} \simeq \sqrt{\mathcal{Q}} \kappa \lambda+\lambda^{2} .
\end{aligned}
$$

The solution at large $\alpha$ would be $R \simeq \kappa \lambda \alpha+R_{0}$ and $\mathcal{D} \simeq \kappa \lambda \alpha+D_{0}$, while $\mathcal{Q}$ is asymptotically given through the implicit equation

$$
\sqrt{\mathcal{Q}} \simeq \frac{1}{2} \kappa \lambda \alpha+\frac{\lambda}{\kappa} \ln (\sqrt{\mathcal{Q}} \kappa+\lambda)+\frac{1}{2} \kappa \lambda \kappa_{0} .
$$

Here $R_{0}, D_{0}$, and $\kappa_{0}$ are integration constants. Hence, asymptotically, $\sqrt{\mathcal{Q}} \sim \frac{1}{2} \kappa \lambda \alpha$ which is 
incompatible with the requirement of good generalization $\mathcal{R} / \sqrt{\mathcal{Q}} \rightarrow 1$. Thus the algorithm will not converge, if $\lambda$ is kept fixed.

The question arises, however, whether a simple extension of the algorithm may not overcome the Odyssean dilemma hinted at in the beginning of this section. We hence suggest to tune the parameter $\lambda$ such that it is large enough at small $\alpha$ to overcome the pre-asymptotic conditions and it tends to zero at large $\alpha$ in order to approach asymptotically the perceptron rule. As can be seen on Fig. 11 this procedure seems successful.

Since the situation is now much more complicated we shall not try to solve the general asymptotic problem, as we did in the case of non-structured data, but we shall limit ourselves to prove that robust solutions exist. For this we start with the following ansatz:

$$
\begin{aligned}
\lambda & =\lambda_{0} \alpha^{-r}, \\
\mathcal{Q} & =c^{2} \alpha^{2 q}, \\
\epsilon_{g} & =a \alpha^{-p}, \\
\omega & =b \alpha^{-s} .
\end{aligned}
$$

with $\omega$ defined via (20). The asymptotic equations obtained from the flow equations (17)-(19) assuming $p \sim q \sim r>s \geq 0$ are of the form:

$$
\begin{aligned}
2 \sqrt{\mathcal{Q}} \frac{d \epsilon_{g}}{d \alpha} & \simeq A_{11} \lambda+\frac{A_{12}}{\sqrt{\mathcal{Q}}}+A_{2} \epsilon_{g} \\
2 \sqrt{\mathcal{Q}} \sin \frac{z}{2} \epsilon_{g} \frac{d \omega}{d \alpha} & \simeq B_{11} \lambda+\frac{B_{12}}{\sqrt{\mathcal{Q}}}+B_{2} \epsilon_{g} \\
\frac{d \sqrt{\mathcal{Q}}}{d \alpha} & \simeq C_{0} \lambda+C_{1} \epsilon_{g}
\end{aligned}
$$

Here the coefficients $A_{\gamma}, B_{\gamma}, C_{\gamma}$ are function of $m, z, L$ and of $\omega$ (the explicit expressions obtained by Maple are given in Appendix, section 5.2).

It is easy to see that an asymptotic solution can exist for:

$$
p=q=r=1 / 2, \quad s=0,
$$

which is therefore compatible with the assumptions used to derive the asymptotic equations (30)-(32). Then $a, b, c$ are obtained as function of $m, \eta, L$ for given $\lambda_{0}$, with some restrictions on the latter (notice that the coefficients $A_{\gamma}, B_{\gamma}, C_{\gamma}$ depend nonlinearly on $\omega$, hence on $b$ ). For illustration, we show in Fig. 2 2 the values of $a, b$ and $c$ as function of $\lambda_{0}$ for $L=10, m=1$ and two values of the data-teacher overlap $\eta$. Notice that there is no asymptotic solution for $\lambda_{0}$ below $\simeq 0.2$.

In Fig. 3 we show the solution of the full equations (17)-(19) - compare also with Fig. 田 which can be seen to approach the asymptotic solution (26)-(33). The solutions are robust in the sense that for all $m, \eta, L$ there exists a large region of $\lambda_{0}$ leading to convergence according to (33). Notice, however, that in the pre-asymptotic region similar phenomena to the non-structured data case seem to take place: the flow is divided by a separatrix defined by a $\lambda_{0, c}$ (the MC simulation presents the same effect, see Fig. 1). 


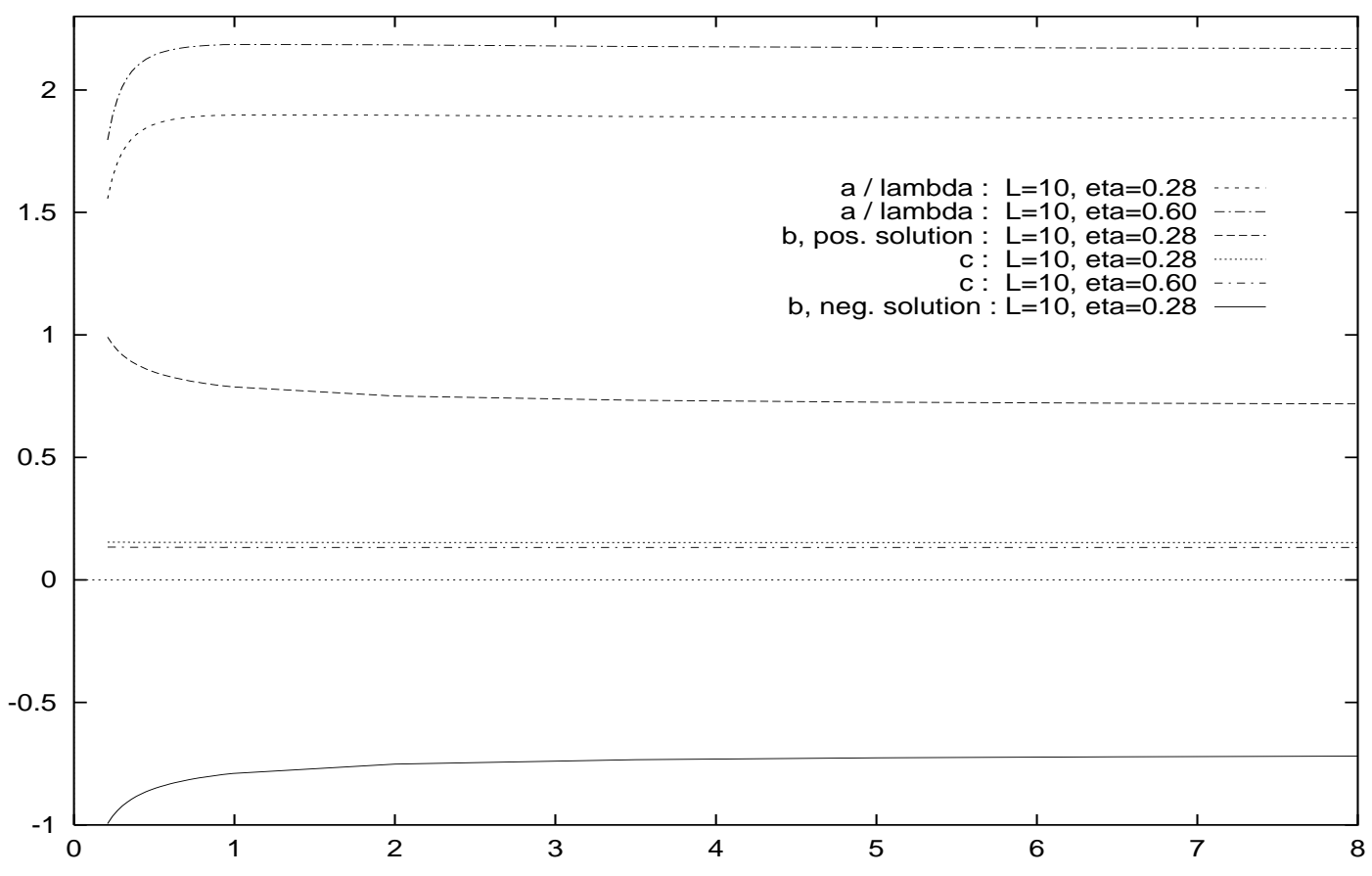

Figure 2: Asymptotic solution (20)-(33) for $L=10: a / \lambda_{0}, b$ and $c$ as function of $\lambda_{0}$ for two values of the overlap, $\eta=0.28,0.6$. There are generally two solutions $a, \pm b, c$ with $b$ practically independent on $\eta$. Note that there is no solution for $\lambda_{0}<\lambda_{0, c}^{\text {asympt. }} \sim 0.2$.

We have thus shown that the simple decrease of $\lambda$ as $1 / \sqrt{\alpha}$ provides convergence to asymptotic perfect generalization with the power $-1 / 2$. Alternatively, one can decrease $\lambda$ as $1 / \sqrt{\mathcal{Q}}$, or as $e(\alpha)$, where

$$
e(\alpha)=\frac{1}{\alpha} \sum_{q=1}^{\alpha N / L} e_{q}
$$

using the running "observed error" $e_{q}$ (2) (this is in a sense the most natural choice, since the student only applies its observations). Again the algorithm is stable against noise or a further dilution of the information introduced by taking $w<1$. See Fig. 1 .

\section{Summary and Discussion}

In the present paper we have investigated the performance of the AR-Hebb-algorithm introduced in [4] in the case where the input patterns are structured. The pattern statistics is characterized by the anisotropy vector $m \boldsymbol{C}$ and performance of the learning rule depends on $m$ and on the overlap $\eta$ between the anisotropy vector and the vector $\boldsymbol{B}$ that defines the rule - apart from the parameters $\lambda$ and $L$ which characterize the AR-Hebb-algorithm. 

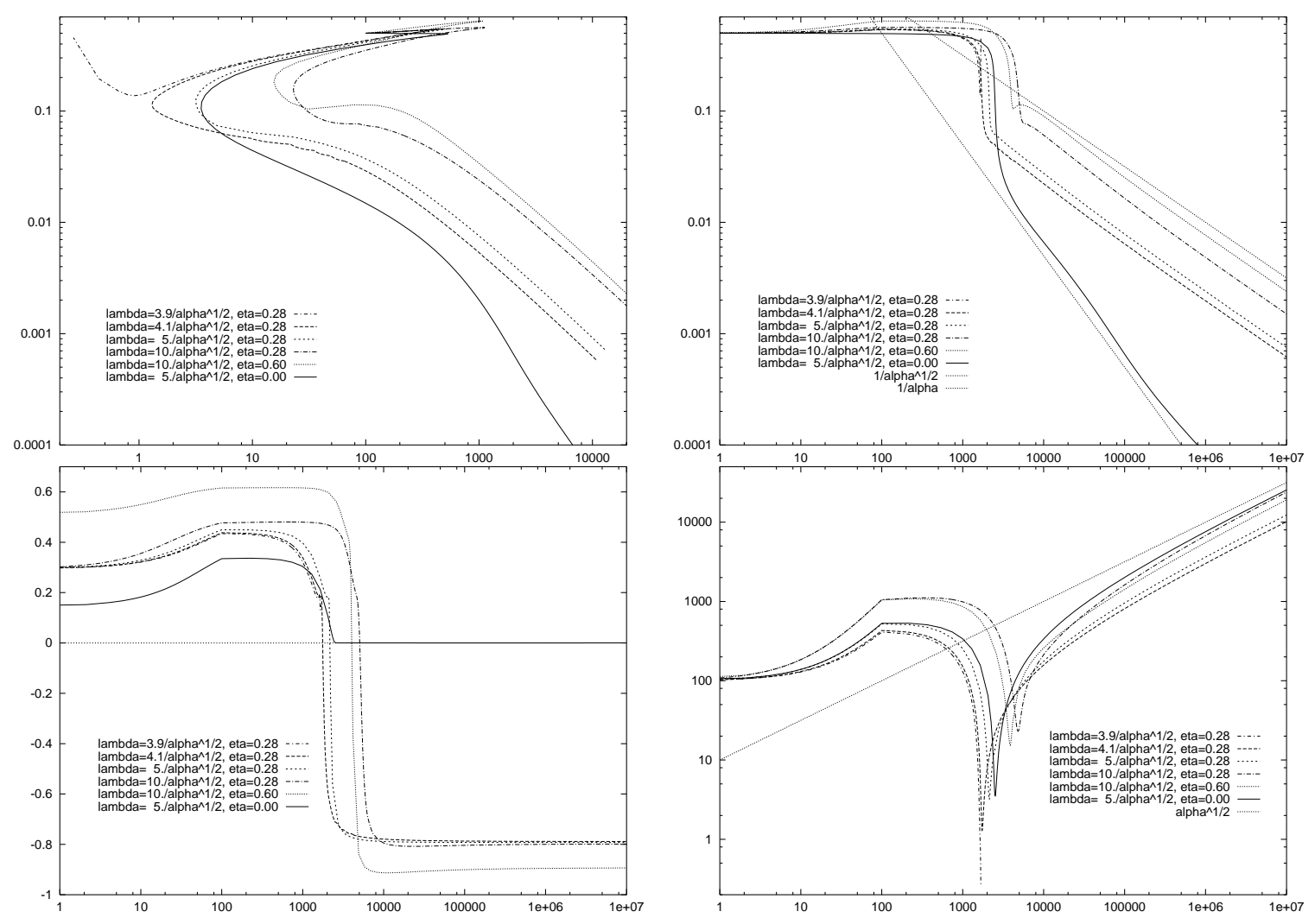

Figure 3: Solution of the flow equations $\sqrt{17)}-(19)$ for $L=10$, starting point $\sqrt{\mathcal{Q}(0)}=100$ and various overlaps $\eta$ : flow with $\alpha$ in the $\epsilon_{g}-\sqrt{\mathcal{Q}}$ plane (upper left), $\epsilon_{g}$ vs $\alpha$ (upper right), $\omega$ vs $\alpha$ (lower left) and $\sqrt{\mathcal{Q}}$ vs $\alpha$ (lower right). Note the change in behaviour between $\lambda_{0}=3.9$ and $\lambda_{0}=4.1$, compare with Fig. . .

As for usual Hebb learning, a tuning of learning parameters is required to achieve good generalization for the classification of structured patterns. Given $L$, the only free parameter of the algorithm is $\lambda$, and tuning of $\lambda$ may proceed in various ways. For instance, one may scale $\lambda$ either with $\alpha$, i. e., with the number of input-output pairs presented, or with the self-overlap $\mathcal{Q}$, or with the empirical error-rate $e_{q}$. Our analysis reveals that the scaling $\lambda \sim \alpha^{-1 / 2}$, which according to that analysis is equivalent to the scalings $\lambda \sim \mathcal{Q}^{-1 / 2}$, or $\lambda \sim e(\alpha)$, leads to asymptotically perfect generalization. The behaviour is robust in the sense that the prefactor $\lambda_{0}$ may be varied over a wide range without changing the asymptotic scaling of the generalization error. In this sense the tuning required to obtain a working algorithm is not fine-tuning. The only requirement for obtaining good generalization is that $\lambda_{0}$ in (26) exceeds a certain minimum value, $\lambda_{0, c}^{a s y m p t}$. This behaviour is reminiscent of the fact that a minimum value of $\lambda$ was also required in the case of unstructured data. In that case, however, the reason was entirely related to pre-asymptotic 
behaviour related to the fixed-point structure of the flow equations, whereas the above analysis is restricted to the asymptotic domain.

From the numerical solution of the full flow-equations and from simulations, we see some empirical evidence that a non-trivial fixed point structure governing the pre-asymptotic behaviour in analogy to what has been found in [4 is present also in the case studied here. As the present dynamical problem is three-dimensional instead two-dimensional, however, the consequences of this might be suspected to be less severe. For instance, a fixed-point with stable and unstable directions in three dimensions does not necessarily produce a separatrix as in the two-dimensional case. However, the projection onto the $\epsilon_{g^{-}} \sqrt{\mathcal{Q}}$ plane shows a separatrix and hence a $\lambda_{0, c}$, as in the unstructured data case (see Fig. 3), with $\lambda_{0, c}>\lambda_{0, c}^{\text {asympt. }}$. Unlike in the two-dimensional case with unstructured patterns, we have so far not found any evidence of non-universal behaviour of the generalization curve. Whether this is intrinsically related to the different role fixed points appear to play in the present case, we do at present not know. Exceptional behaviour appears for $\eta=0$ which in the student-teacher scenario, however, is equivalent to the unstructured case.

Acknowledgments: This project was initiated during the Seminar 'Statistical Physics of Neural Networks' in Dresden, March 1999. The authors would like to thank the Max Planck Institut für Physik Komplexer Systeme in Dresden for hospitality and financial support and the participants to the workshop for interesting discussions.

\section{Appendix}

\subsection{Expectations values}

The expectations values $A$., $S$. in (17)-(19) are:

$$
\begin{aligned}
A_{J T} & =m \eta \varphi\left(m \frac{\mathcal{D}}{\sqrt{\mathcal{Q}}}\right)+\sqrt{\frac{2}{\pi}} \frac{\mathcal{R}}{\sqrt{\mathcal{Q}}} \mathrm{e}^{-\frac{m^{2} \mathcal{D}^{2}}{2 \mathcal{Q}}} \\
& =m \cos z \varphi(m \cos y)+\sqrt{\frac{2}{\pi}} \cos x \mathrm{e}^{-\frac{1}{2} m^{2} \cos ^{2} y}, \\
A_{T T} & =m \eta \varphi(m \eta)+\sqrt{\frac{2}{\pi}} \mathrm{e}^{-\frac{m^{2} \eta^{2}}{2}} \\
& =m \cos z \varphi(m \cos z)+\sqrt{\frac{2}{\pi}} \mathrm{e}^{-\frac{1}{2} m^{2} \cos ^{2} z}, \\
S_{J T} & =1+\varphi\left(m \frac{\mathcal{D}}{\sqrt{\mathcal{Q}}}\right)-\varphi(m \eta)-4 G\left(\frac{\mathcal{R}}{\sqrt{\mathcal{Q}}}, \frac{\mathcal{D}}{\sqrt{\mathcal{Q}}}, \eta\right) \\
& =1+\varphi(m \cos y)-\varphi(m \cos z)-4 G(\cos x, \cos y, \cos z), \\
A_{J J} & =m \frac{\mathcal{D}}{\sqrt{\mathcal{Q}}} \varphi\left(m \frac{\mathcal{D}}{\sqrt{\mathcal{Q}}}\right)+\sqrt{\frac{2}{\pi}} \mathrm{e}^{-\frac{m^{2} \mathcal{D}^{2}}{2 \mathcal{Q}}} \\
& =m \cos y \varphi(m \cos y)+\sqrt{\frac{2}{\pi}} \mathrm{e}^{-\frac{1}{2} m^{2} \cos ^{2} y}, \\
A_{T J} & =m \frac{\mathcal{D}}{\sqrt{\mathcal{Q}}} \varphi(m \eta)+\sqrt{\frac{2}{\pi}} \frac{\mathcal{R}}{\sqrt{\mathcal{Q}}} \mathrm{e}^{-\frac{m^{2} \eta^{2}}{2}}
\end{aligned}
$$




$$
\begin{aligned}
& =m \cos y \varphi(m \cos z)+\sqrt{\frac{2}{\pi}} \cos x \mathrm{e}^{-\frac{1}{2} m^{2} \cos ^{2} z}, \\
A_{J C} & =m \varphi\left(m \frac{\mathcal{D}}{\sqrt{\mathcal{Q}}}\right)+\sqrt{\frac{2}{\pi}} \frac{\mathcal{D}}{\sqrt{\mathcal{Q}}} \mathrm{e}^{-\frac{m^{2} \mathcal{D}^{2}}{2 \mathcal{Q}}} \\
& =m \varphi(m \cos y)+\sqrt{\frac{2}{\pi}} \cos y \mathrm{e}^{-\frac{1}{2} m^{2} \cos ^{2} y}, \\
A_{T C} & =m \varphi(m \eta)+\sqrt{\frac{2}{\pi}} \eta \mathrm{e}^{-\frac{m^{2} \eta^{2}}{2}} \\
& =m \varphi(m \cos z)+\sqrt{\frac{2}{\pi}} \cos z \mathrm{e}^{-\frac{1}{2} m^{2} \cos ^{2} z},
\end{aligned}
$$

where

$$
\begin{aligned}
G\left(\frac{\mathcal{R}}{\sqrt{\mathcal{Q}}}, \frac{\mathcal{D}}{\sqrt{\mathcal{Q}}}, \eta\right) & =\frac{1}{2} \int_{-\infty}^{m \frac{\mathcal{D}}{\sqrt{\mathcal{Q}}}} \frac{d t}{\sqrt{2 \pi}} \mathrm{e}^{-\frac{1}{2} t^{2}}\left(1+\varphi\left(\frac{t \frac{\mathcal{R}}{\sqrt{\mathcal{Q}}-m \eta}}{\sqrt{1-\frac{\mathcal{R}^{2}}{\mathcal{Q}}}}\right)\right) \\
& =\frac{1}{2} \int_{-\infty}^{m \cos y} \frac{d t}{\sqrt{2 \pi}} \mathrm{e}^{-\frac{1}{2} t^{2}}\left(1+\varphi\left(\frac{t \cos x-m \cos z)}{\sin x}\right)\right) \\
\varphi(x) & =\operatorname{erf}(x / \sqrt{2})
\end{aligned}
$$

with erf the error function.

\subsection{Asymptotic coefficients}

The Maple expressions for the coefficients $A_{\gamma}, B_{\gamma}, C_{\gamma}$ in (30)-(32) are:

$$
\begin{aligned}
u & =\frac{1}{2} m \cos (z) \sqrt{2} \\
v & =m \sin \left(\frac{1}{2} z\right) \sqrt{2} \\
g(v \omega) & =v \omega \operatorname{erf}(v \omega) \\
f(v \omega) & =v \omega \operatorname{erf}(v \omega)+\frac{e^{-v^{2} \omega^{2}}}{\sqrt{\pi}} \\
A_{11} & =-4 \frac{\sin \left(\frac{1}{2} z\right) \operatorname{erf}(u) \omega}{\pi} \\
A_{12} & =\frac{e^{-u^{2}} f(v \omega)}{\pi \sqrt{\pi} L} \\
A_{2} & =\frac{e^{-u^{2}}\left(-2 \frac{1+2 v^{2} \omega^{2}}{L}+4\left(1-\frac{1}{L}\right) \operatorname{erf}(u) f(v \omega)\right)}{\sqrt{2 \pi}} \\
B_{11} & =4 \frac{m \sin \left(\frac{1}{2} z\right)^{2} \operatorname{erf}(u)\left(\omega^{2}-1+s^{2}\right)}{\pi} \\
B_{12} & =-\frac{\sin \left(\frac{1}{2} z\right) e^{-u^{2}} \omega f(v \omega)}{\pi^{3 / 2} L}
\end{aligned}
$$




$$
\begin{aligned}
& B_{2}=4 \frac{m \sin \left(\frac{1}{2} z\right)^{2} e^{-u^{2}}\left(\omega^{2}-1+s^{2}\right)\left(\frac{v \omega}{L}-\left(1-\frac{1}{L}\right) \operatorname{erf}(u) f(v \omega)\right)}{\sqrt{\pi}} \\
& C_{0}=\sqrt{2} u \operatorname{erf}(u)+\frac{\sqrt{2} e^{-u^{2}}}{\sqrt{\pi}} \\
& C_{1}=-\left(1-\frac{1}{L}\right) e^{-u^{2}} f(v \omega)\left(\sqrt{\pi} m \operatorname{erf}(u) \cos (z)+\sqrt{2} e^{-u^{2}}\right)
\end{aligned}
$$

\section{References}

[1] Watkins, C.J.C.H. (1989), "Learning from delayed rewards", Ph.D.Thesis, unpublished.

[2] Sutton, R.S. (1988), Machine Learning 3, 9.

[3] Mlodinow, L. and Stamatescu, I.-O. (1985), Int. J. of Comp. and Inform. Sci, 14, 201.

[4] Kühn, R. and Stamatescu, I.-O. (1999), J. Phys. A: Math. Gen. 32, 5479.

[5] Riegler, P., Biehl, M., Solla S.A. and Marangi, C (1996) in Proceedings of the 7th Italian Workshop on Neural Networks 1995, eds. Marinaro, M. and Tagliaferr, R., p. 87, World Scientific (Singapore).

[6] Biehl, M. and Riegler, P. (1994), Europhys. Lett. 28, 525.

[7] Vallet, F. (1989), Europhys. Lett. 8, 747.

[8] Hertz, J., Krogh, A. and Palmer, R.G. (1991), Introduction to the Theory of Neural Computation (Addison-Wesley, Reading, Mass.).

[9] Kinouchi, O. and Caticha, N. (1992), J. Phys. A 25, 6243.

[10] Biehl, M. and Schwarze, H. (1992), Europhys. Lett. 20, 733. 